\title{
Constraining wind clumping and mass-loss in OB stars with COBRaS: The e-MERLIN Cyg OB II Radio Survey
}

Jack Morford*

Department of Physics \& Astronomy, University College London, UK.

E-mail: jmorfordestar.ucl.ac.uk

\section{Danielle Fenech}

Department of Physics \& Astronomy, University College London, UK.

E-mail: dmfestar.ucl.ac.uk

\section{Raman Prinja}

Department of Physics \& Astronomy, University College London, UK.

Email: rkp@star.ucl.ac.uk

\begin{abstract}
Luminous, massive stars provide one of the greatest feedback mechanisms of energy and material back into the interstellar medium (ISM), providing 'food' for the ever on-going star formation from galaxy to galaxy. An important parameter in desperate need of pinning down is the rate at which OB stars lose mass, such that we obtain an accurate picture of massive star stellar winds. Strong evidence suggests that previous mass-loss rates have been over-estimated and descrepancies between different mass-loss diagnostics excite the need for structured stellar wind models on both small (clumping) and large (co-rotating interaction regions) scales. COBRaS, the eMERLIN Legacy Cyg OB II Radio Survey shall deliver high resolution, deep field maps at both L- and C-band to deliver accurate mass-loss estimates of a large sample of O and B stars. In manufacturing the most substanstial radio dataset of the Cyg OB II region, COBRaS is expected to clarify our understanding of massive star winds and constrain clumping (small-scale structure) by probing the outer region of $\mathrm{OB}$ stellar winds.
\end{abstract}

12th European VLBI Network Symposium and Users Meeting

7-10 October 2014

Cagliari, Italy

\footnotetext{
* Speaker.
} 


\section{An introduction to massive star winds}

Massive stars play a crucial role in shaping our universe, recycling energy and material back into the inter-stellar medium (ISM) and driving star formation. The key parameter in describing a hot star wind is its mass-loss rate, dictating the star's evolution and fate throughout its life time. For many years it was suggested that accurate mass-loss determinations of OB stars were being over-estimated and at present there exists strong evidence that this is indeed the case [8]. This suggestion and the discordance between values obtained via different means [2] have led to a revised theory of massive star stellar winds. In the past, OB star winds were thought to be smooth and homogeneous, now theories have moved into the realms of structured wind models. Radiative line driving provides the mechanism behind stellar wind outflows and it is predicted that small-scale density inhomogeneities in the inner wind are driven outwards and expand to create 'clumps' of enhanced density whilst the inter-clump medium is that of a lower density [8]. This 'clumping' effect is thought to account for the over-estimates of previous mass-loss estimates from massive stars. However its scale and evolution throughout the winds of hot stars has yet to have been properly constrained.

Several ways in which to derive a massive star's mass-loss rate exist. Namely, using UV P-Cygni line profiles, measuring the thermal radio or FIR continuum emission or by modelling the $\mathrm{H} \alpha$ line using NLTE (non-local thermodynamic equilibrium) atmosphere codes [8]. The later two methods are both $\rho^{2}$ diagnostics and are strongly dependant upon the density of the emitting region - strongly sensitive to clumping. Mass-loss rates inferred from UV P-Cygni line profiles are imperceptive to clumping, depending purely on the abundance of the element in question and its ionisation fraction [4].

\section{Small and large scale structure in the winds of massive stars}

Clumping describes small-scale structure whereby initial small-scale density inhomogeneities within the inner wind (close to the photosphere) [1] re-distribute matter into enhanced density clumps we shall call $\rho(H)$. Instabilities within the radiative line-driving mechanism cause these clumps to grow, whilst the density of the inter-clump gas $\rho(L)$ falls [7]. Motivated from hydrodynamical simulations of massive star winds that include this line-driven instability, the interclump gas is assumed to be a vacuum [9]. The clumping factor as described by Owocki [6] goes as:

$$
f_{c l}=<\rho^{2}>/<\rho>^{2}
$$

and is a measure of the over density within the clumps. Their effect also leads to an enhanced opacity, which for $\rho^{2}$ dependant processes and hence diagnostics, the mass-loss rates are reduced by a factor of $\sqrt{f_{c l}}$. Thus clumping provides a solution to previous found mass-loss rates that are thought to be over-estimates of the true value. Owocki, [7] suggested that it is possible for the enhanced density clumps $\rho(H)$ to become optically thick, in which a porosity effect must be introduced $h=L^{3} / l^{2}$ where 1 is the size and L the seperation of the clumps. In this sense, photons won't escape the optically thick clumps meaning the size and shape of the clumps becomes important. 
Large scale structure also exists within the winds of massive stars, namely Co-rotating Interaction Regions (CIRs). The UV lines of various $\mathrm{O}$ stars have been found to show periodic variability over time-scales larger than a day. It is thought they originate from large regions of enhanced wind flux from the stellar surface [5], which sweeps out as the star rotates. Although mass-loss estimates are not directly altered by the CIRs when using the radio free-free emission diagnostic, their presence must be considered when comparing results from the UV P-Cygni line diagnostic.

\section{Mass-loss estimates in the radio regime}

Radio emission from massive star winds, radiates from the outermost part of the wind, typically between 10 and $100 R_{*}$. The recieved radio flux is due to free-free emission and hence scales with the density of the emitting material squared. As mentioned above, this is a critical feature of radio observations of massive stars since its strong sensitivity to any clumping present within the wind [8]. In comparison to FIR wavelengths, radio fluxes are considered the 'cleaner' since the winds become optically thick in these regions $\left(\geq 10 R_{*}\right)$, the wind has reached its terminal velocity and is no longer accelerating, such that the $\rho_{\text {wind }}$ is independant of the wind velocity, $v(r)$. The amount of mass lost from the star is then simply obtained using the equation:

$$
\dot{M}=0.095 \frac{\mu v_{\infty} S_{v}^{3 / 4} D^{3 / 2}}{Z \gamma^{1 / 2} g^{1 / 2} v^{1 / 2}} M_{\odot} / y r
$$

as found by the work of [10]. Although this equation holds for a smooth wind model, it can be easily altered to account for a structured, 'clumped' wind by the introduction of the clumping factor (equation 2.1). Furthermore, no photospheric correction or complex NLTE atmosphere codes are required as is the case for the $\mathrm{H} \alpha$ mass-loss diagnostic. It is a straight forward mass-loss dianostic that is sensitive to clumping and directly probes the outer region of a massive star's stellar wind. Many O stars infact have companions, in the Cyg OB II associtation, its binary fraction is estimated to be 55\% [3]. In the case of a binary system, it is somewhat likely that the free-free radio emission will be non-thermal due to colliding winds between the binary pair [4]. Therefore one must have multi-frequency observations in order to find the spectral index of the source in question to discover if this is the case.

\section{COBRaS - The e-MERLIN Cyg OB II Radio Survey}

COBRaS will deliver accurate flux measurements for a large number of OB stars, for further information on the project, see Fenech et al. PoS (EVN 2014). With the use of equation 3.1 (modified to include the clumping factor, $f_{c l}$ ), accurate mass-loss rates can be estimated for the identified OB stars whilst constraints can be built upon the clumping factor. In combination with (incoming) $H \alpha$ and sub-millimetre data, we will further our model of a massive star wind, constraining clumping as a function of stellar radii. The dual-radio bands of the COBRaS data will also provide a unique opportunity to study the incidence of non-thermal emission from within the stellar cluster. Any detected non-thermal emission will suggest a massive star binary system, allowing for an updated estimate of the binary fraction within the Cyg OB II association. In conjunction with other multi-waveband surveys of the Cygnus X region (IPHAS, Spitzer, Chandra), COBRaS will yield 
substantial results for the key science areas mentioned above as well as providing new propectives for additional archival studies.

COBRaS C-band observations will be crucial to the mass-loss study of OB stars within Cyg OB II, with the majority of observing time being awarded at C-band. Observations are due to commence at the beggining of 2015. Calibration of the Legacy L-band dataset is currrently in progress and we hope to have reduced and imaged the entire 42 hours by the start of 2015 (please refer to Fenech et al. PoS(EVN 2014), for information regarding the current status of the L-band observations). Aiming to achieve sensitivities down to $8 \mu \mathrm{Jy} / \mathrm{beam}$, we are highly expectant to pick out $\mathrm{O}$ and $\mathrm{B}$ star fluxes having estimated the 6 and $20 \mathrm{~cm}$ fluxes from a collated catalogue of the known massive stars within our seven pointings.

\section{References}

[1] D. C. Abbott, J. H. Bieging, and E. Churchwell. Mass loss from very luminous OB stars and the Cygnus superbubble. apj, 250:645-659, November 1981.

[2] A. W. Fullerton, D. L. Massa, and R. K. Prinja. The Discordance of Mass-Loss Estimates for Galactic O-Type Stars. apj, 637:1025-1039, February 2006.

[3] H. A. Kobulnicky, D. C. Kiminki, M. J. Lundquist, J. Burke, J. Chapman, E. Keller, K. Lester, E. K. Rolen, E. Topel, A. Bhattacharjee, R. A. Smullen, C. A. Vargas Alvarez, J. C. Runnoe, D. A. Dale, and M. M. Brotherton. Toward Complete Statistics of Massive Binary Stars: Penultimate Results from the Cygnus OB2 Radial Velocity Survey. ArXiv e-prints, June 2014.

[4] D. Massa, A. W. Fullerton, and R. K. Prinja. P V and the Mass Loss Discrepancy in O Stars. In G. Sonneborn, H. W. Moos, and B.-G. Andersson, editors, Astrophysics in the Far Ultraviolet: Five Years of Discovery with FUSE, volume 348 of Astronomical Society of the Pacific Conference Series, page 118, June 2006.

[5] D. Massa, L. Oskinova, A. W. Fullerton, R. K. Prinja, D. A. Bohlender, N. D. Morrison, M. Blake, and W. Pych. CIR modulation of the X-ray flux from the O7.5 III(n)((f)) star $\xi$ Persei. mnras, 441:21732180, July 2014.

[6] S. P. Owocki, J. I. Castor, and G. B. Rybicki. Time-dependent models of radiatively driven stellar winds. I - Nonlinear evolution of instabilities for a pure absorption model. apj, 335:914-930, December 1988.

[7] S. P. Owocki, K. G. Gayley, and N. J. Shaviv. A Porosity-Length Formalism for Photon-Tiring-limited Mass Loss from Stars above the Eddington Limit. apj, 616:525-541, November 2004.

[8] J. Puls, N. Markova, and S. Scuderi. Stellar winds from massive stars - What are the REAL mass-loss rates? ArXiv Astrophysics e-prints, July 2006.

[9] J. Puls, J. S. Vink, and F. Najarro. Mass loss from hot massive stars. aapr, 16:209-325, December 2008.

[10] A. E. Wright and M. J. Barlow. The radio and infrared spectrum of early-type stars undergoing mass loss. mnras, 170:41-51, January 1975. 